\title{
Exoskeleton Technology in Rehabilitation: Towards an EMG-Based Orthosis System for Upper Limb Neuromotor Rehabilitation
}

\author{
Luis Manuel Vaca Benitez, ${ }^{1}$ Marc Tabie, ${ }^{2}$ Niels Will, ${ }^{1}$ Steffen Schmidt, ${ }^{1}$ \\ Mathias Jordan, ${ }^{1}$ and Elsa Andrea Kirchner ${ }^{1,2}$ \\ ${ }^{1}$ Robotics Innovation Center, German Research Center for Artificial Intelligence (DFKI), Robert-Hooke-Straße 5, \\ Bremen, Germany \\ ${ }^{2}$ Robotics Lab, University of Bremen, Robert-Hooke-Straße 5, Bremen, Germany
}

Correspondence should be addressed to Luis Manuel Vaca Benitez; luis.vaca_benitez@dfki.de

Received 19 July 2013; Accepted 14 October 2013

Academic Editor: Kazuhiko Terashima

Copyright (c) 2013 Luis Manuel Vaca Benitez et al. This is an open access article distributed under the Creative Commons Attribution License, which permits unrestricted use, distribution, and reproduction in any medium, provided the original work is properly cited.

\begin{abstract}
The rehabilitation of patients should not only be limited to the first phases during intense hospital care but also support and therapy should be guaranteed in later stages, especially during daily life activities if the patient's state requires this. However, aid should only be given to the patient if needed and as much as it is required. To allow this, automatic self-initiated movement support and patientcooperative control strategies have to be developed and integrated into assistive systems. In this work, we first give an overview of different kinds of neuromuscular diseases, review different forms of therapy, and explain possible fields of rehabilitation and benefits of robotic aided rehabilitation. Next, the mechanical design and control scheme of an upper limb orthosis for rehabilitation are presented. Two control models for the orthosis are explained which compute the triggering function and the level of assistance provided by the device. As input to the model fused sensor data from the orthosis and physiology data in terms of electromyography (EMG) signals are used.
\end{abstract}

\section{Introduction}

The requirements on a social, well-functioning, and modern health care system-including elderly care-are demanding: it must be flexible enough to encounter the increasing process of change and the related challenges. These changes and challenges are triggered, among other things, by the demographic changes, the increase in chronic diseases, the rising costs, and the impending skills shortage [1].

To assure the achievement of these objectives in medical care, the publicly financed science plays a major role. In this context, robotics research is an important element which is increasingly gaining significance [2].

Nowadays, robotic systems are used in various medical disciplines and different highly specialized applications, for example, in the field of minimally invasive surgery [3]. Furthermore, technical therapy approaches in physiotherapy and occupational therapy are given more and more importance.
In this context, particular assistance and training devices are in the center of interest. These could be systems like powered exoskeletons, active orthoses, or special end-effector-based therapy robots [4]. On the one hand, these systems could provide important support in medical rehabilitation for the therapist and patient and, on the other hand, they could be a help in everyday activities for the elderly or motor-impaired people in their home environment [5].

Due to the aging society and probably significant increase in chronic diseases of the musculoskeletal and the nervous system, the need for innovation in assistive technologies for everyday activities and rehabilitation is judged as very high [6].

In general, independent living and acting are strongly connected with the motor skills of the individual. The proper function of the arm and hand in everyday activities - at work or at home-are of vital importance [7]. 
In the long-term view, our superior goal is to design and develop a full home rehabilitation system, composed of an exoskeleton and physiological data acquisition and processing in terms of EEG, EMG, and gaze-direction, which can be operated in real as well as in virtual environments. For this purpose we will make use of our acquired expertise, gained with the development of exoskeleton systems in recent projects [8]. The exoskeleton shall be lightweight and comfortable to wear, while having enough force to move both plegic arms and the upper body of a patient. With the use of physiological data movement intentions of the patient can be predicted and therefore the interaction between subject and rehabilitation device can be improved. The system is meant to support a therapist in the daily routines during the rehabilitation phase of a patient. When using the system in a virtual environment it is possible for the therapist to design and change tasks for the patient without being on sight and in addition the training/rehabilitation success can be monitored via physiological data, for example, EMG signals.

Anyhow, in the first step we decided to design and develop an orthotic system which accounts with one active degree of freedom (DOF) capable of flexing and extending a patient's arm in parallel with the upper body exoskeleton. This gives us the opportunity of analyzing and developing physiological data driven control strategies for an exoskeleton in an easierto-handle setup compared to full upper body system.

Therefore, in this paper we introduce the concept and medical background of a support and rehabilitation system for the upper limb in the form of an active elbow orthosis.

A demonstrator of the orthosis is developed and driven in terms of EMG signals from the upper arm measured from the biceps brachii and triceps brachii [9]. The control is based on threshold functions which are correlated to maximum amplitudes measured in both muscles.

In this paper we conduct experiments to improve the control of the system. We derived a model with the Recursive Least Square (RLS) algorithm which takes into account EMGs from the upper arm as well as sensor data from the orthosis (position and force induced to the device). Off-line gained results for the triggering of the orthosis are provided. In addition, we present results of an experiment giving the orthosis the ability to adjust the level of support provided to the patient. Again a model is formulated with the RLS involving the same input data. Having the possibility of automatic support adjustment during rehabilitation can have positive effects since an increasing muscle activity results in a lower support. In this way the support is constantly reduced until the point the patient does not need any external help for moving his arm.

Furthermore, by means of the movement prediction, the patient has the impression to control the arm himself, although the orthosis is actually moving the arm. This reconnects the movement planning phase of the brain with movement execution to reestablish the capability of the patient for free and self-paced movements. Therefore, the combination of the self-initiated movement support and patient-cooperative control strategies can lead to a positive effect on rehabilitation and user-centered support in daily activities.
In the long term, this device could be used for the entire rehabilitation process, for example, to improve motor recovery in patients with neurological or orthopaedic lesions. Furthermore, the progress of therapy can be evaluated by monitoring and analyzing the muscle activity via EMG.

\section{Use of Robots in Rehabilitation}

In this section, the basic elements of rehabilitation robotics are presented. This includes the medical background for rehabilitation, the basic functions of robotic systems and applications, target groups, and transferable treatment modalities which can be suited to adapt on rehabilitation devices.

2.1. Foundations of Rehabilitation of Impairments of the Motor System. Common causes of acquired and permanent physiological defects such as limited motor skills are mostly neurological diseases or injuries. In this case, one of the most common causes of permanent disabilities in western civilization is stroke [10]. Only about $40 \%$ of all stroke survivors are able to return to normal employment and onethird are permanently dependent on support and care. Hence, the main goal of rehabilitation is the reintegration of affected people back into normal life in an optimal way [11].

In general, rehabilitation can achieve its goals in two ways: through compensation of motor dysfunction and/or through recovery of motor functions. Here, a force exerting exoskeleton or orthosis for rehabilitation applications is used for compensation of motor deficits and/or for the recovery of motor skills. Hereby the extremities with motor deficit-incomplete paralysis (paresis) or with total paralysis (plegia) - are actively supported (e.g., by gravity compensation).

To recover or improve motor function, a preferably early and intensive rehabilitation is recommended, since a positive relationship between treatment intensity and outcome exists [12]. However, this requires a high and efficient deployment of personnel, which can be a limiting factor. In this context, the use of robot-aided therapy is worthwhile [13].

The main problem of a paresis is located in the lack of necessary force, associated with concomitant reduction in range of motion and speed of the affected muscles. Furthermore, the muscle coordination is impaired; this applies to the muscle chain coordination and to the internal muscle coordination. The muscles tend to a prolonged contraction time and a delayed end of the contraction. Moreover, the affected muscles fatigue much faster with respect to the nonaffected muscles [14].

By motor learning the brain is able to adjust to new situations due to massive functional reorganization. This phenomenon is known as neuronal plasticity and characterized by the ability of the brain to restructure itself by forming new neural connections. However, the ability of the brain to restructure itself is limited since it is not plastic in every of its regions. Long-term evaluations will reveal the magnitude of the rehabilitation success. 
2.2. Target Group and Corresponding Applications. For a variety of diseases a motor rehabilitation by means of a robotic device is in general conceivable. We are of the opinion that an application is particularly suitable and economically useful, where long rehabilitation periods are necessary. This applies, for instance, to chronically or chronic-progressive neurological diseases in which a regular, persevering training a rapidly progression of symptoms and sequelae of diseases might be prevented. These are in addition to the surgical and orthopedic diseases, such as elbow and shoulder joint prosthesis, brain tumor surgery, and muscle weakness due to immobilization and surgical follow-up care (mobilization), in essence, the neurological diseases. Examples of such neurological diseases are multiple sclerosis, peripheral nerve lesions, traumatic brain injury, infantile cerebral palsy, and as mentioned stroke.

Stroke is a sudden onset of dysfunction of the motor, sensory, and cognitive functions of the brain. Depending on the location and severity of the injury, the functional limitations may vary.

In the first 3 to 18 days after stroke certain neurotransmitters can be detected. It is believed that these substances are important for the neural plasticity. Therefore, the first weeks are an ideal time to ensure optimal functional and structural reorganization of the brain. Hence, an early active training of the disturbed functions leads to functional recovery [15].

Nevertheless, approximately $35 \%$ of stroke survivors live in the long term with a significant leg paresis and $65 \%$ are not able to use the affected hand in daily activities. To this already high level of suffering, patients often suffer from depression, a so-called poststroke depression. The resulting psychological problems can prevent a successful motor therapy, since the motivation and cooperation of the patient play a crucial role [15].

Hence, a modern rehabilitation of stroke or other neurological diseases is always oriented to the individual patient's condition.

Together with the patient, specialised everyday and (if necessary) job-relevant therapy goals are determined. The treatment team selects an appropriate therapy concept [15]. The various therapeutic measures for arm rehabilitation, which can be transferred to a robotic system, include repetitive training, uni- and bilateral training, the effect of training at distal positions, task-oriented training, and mirror therapy [13, 16-19].

In addition, in the course of treatment the successes or failures are detected and evaluated to adjust the therapeutic measures or to define new therapeutic goals. Thus, the assessment procedures play an important role in motor rehabilitation. Robotic systems which are equipped with assessment functions can make an important contribution to the quality of treatment, since they could ensure a simple and regular review of treatment effects.

By revisiting established therapy measures and assessment methods, robotic therapy systems can be optimally integrated into the rehabilitation process.
2.3. Robotic Systems for Rehabilitation Applications. Exoskeleton or orthotic systems for medical applications in rehabilitation should support scientifically founded training principles. Furthermore, these systems should optimally be integrated into the rehabilitation routine and must support the therapist and patient in a useful way.

The general aims of rehabilitation robotics relate mainly to the increase of efficiency, accuracy, and reproducibility of the treatment methods while ideally improving the economic situation in rehabilitation [20]. In addition to the general objectives, concrete goals have to be formulated. These goals are as follows:

(i) imitation of natural and patient-specific motion,

(ii) high degree of compliance control (this promotes a safe man-machine interaction),

(iii) self-initiated movement support and patientcooperative control strategies (this promotes motor learning processes in the brain),

(iv) solid monitoring of the treatment progress,

(v) early intensive training.

2.3.1. Benefits of Orthosis and Exoskeleton Technology. The properties of an exoskeleton/orthosis offer a number of advantages for use in rehabilitation [21]. The advantages are as follows

(i) Good stabilization and guidance of the limb: compared to end-effector systems (usually with just one interface to the patient) an exoskeleton/orthosis can be connected to several points with the patient. With this specific structure the patient's arm is guided and stabilized at every joint.

(ii) Gravity compensation: the weight of mechanical structures and the human limb can be compensated by different mechanisms.

(iii) Reproduction of the human kinematics with a high number of active degrees of freedom.

(iv) Haptic features: the exoskeleton/orthosis design allows transferring haptic functions at certain points throughout the patient's arm. Possible types of haptic feedback are kinaesthetic feedback (force-feedback) and tactile feedback (e.g., vibrations)

(v) Modular design: exoskeleton/orthosis with a segmental structure allows providing a patient-specific system which meets the requirements and needs of the patient, in the sense that an exoskeleton including only the affected joint would be used. This can reduce costs and deliver a differentiated training, following the slogan: "as much as necessary, as little as possible."

(vi) Force intensification: due to the active degrees of freedom and mobility, an exoskeleton/orthosis can be used as a power amplifier. Through this option, it is possible to deliver a system that supports activities of everyday life and acts in the same time as a training system. 


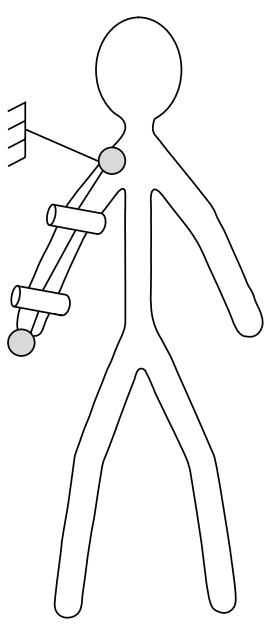

(a)

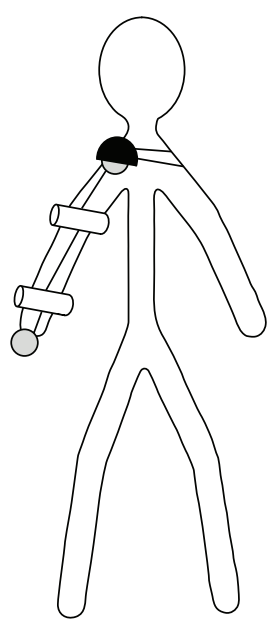

(b)

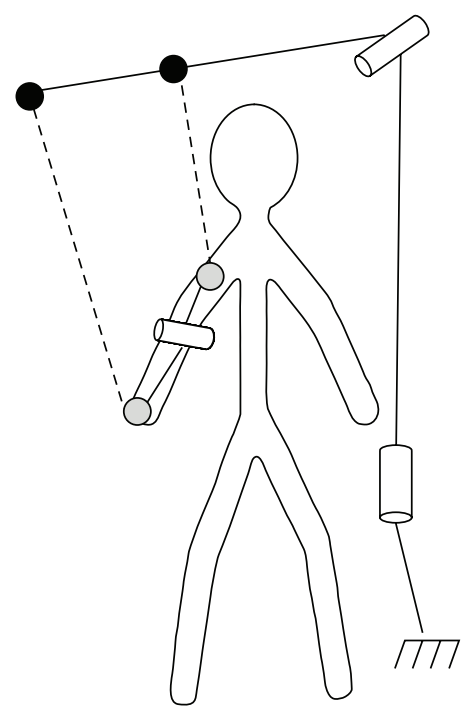

(c)

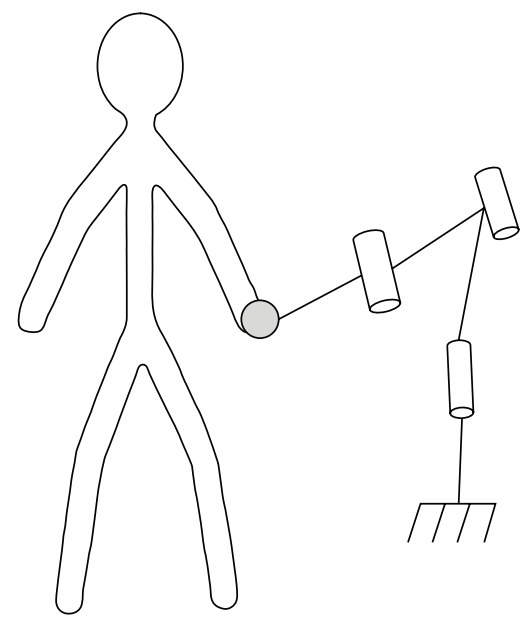

(d)

Figure 1: Mechanical categories of upper limb rehabilitation robots. (a) Semiexoskeleton with fixed base. (b) Mobile exoskeleton (haptic interface). (c) Wire based. (d) End-effector-based.

2.4. Current State of Science and Technology. Robot-aided rehabilitation of upper and lower limbs is currently a fast developing field that is also getting more and more acceptance by clinicians. As mentioned, to recover motor function, an intensive and early rehabilitation is recommended. For this purpose, a large variety of systems which are able to support therapists in their daily work, as well as control approaches, have been developed and are more and more the subject of current research. Furthermore, clinical trials on various systems already show that robotic therapy can be useful and compared to traditional methods of therapy there are no disadvantages in the effectiveness $[22,23]$.

Today, developed or applied robotic rehabilitation systems can be categorized according to their application focus. Depending on the target group (patient's needs), pathology, preferred method of treatment, and place of installation, different conceptual approaches are possible. Rehabilitation systems for the upper limbs can be classified into exoskeleton systems [24, 25] and end-effector-based structures [26, 27] which further divide into uni- and bilateral designs [28], as well as distal and proximal approaches [5, 29]. (In contrast, systems for lower extremity have a greater variety of designs and are already more widely used.) Figure 1 shows the general design of the currently most used systems for upper limb rehabilitation, including portable haptic interfaces for use, for example, in a virtual environment. In majority these systems are equipped with a fixed base and, therefore, are limited in mobility.

All systems offer a so called "massed practice therapy" paradigm but nonetheless the individual systems have, due to their design, benefits, and drawbacks, in common that they are quite focused on their application scenario. (The "massed practice therapy" paradigm involves an intensive, repetitive, frequent, and according to the principles of motor learning oriented practice.) Essentially, restrictions can be found in the range of symptoms, which can be treated, as well as system mobility. Generally, it can be stated that the systems are specialized for particular parameters and there is no system, which fits to all kinds of patients in the same way [13].

For example, the Swiss company Hocoma AG (Industriestrasse $4 \mathrm{CH}-8604$ Volketswil, Switzerland) provides a therapy concept with three different rehabilitation systems for upper limb rehabilitation. This therapy concept is based on taskoriented training scenarios in a virtual environment, which facilitates treatment of neurological diseases of different severity. The three therapeutic robots are ArmeoPower (former ARMin), a robotic arm exoskeleton [30], ArmeoSpring, an exoskeleton with integrated spring mechanism (emerging from T-WREX exoskeleton) [31], and ArmeoBoom, an overhead sling suspension system (emerging from the ROBAR project) [32].

An example for an end-effector-based approach, which is also widely used in modern therapy, is the InMotion Arm Robot (former MIT Manus). This system simulates the classical hand-to-hand therapy of a therapist with a continuous measurement of position and force applied to the arm of the patient. It is also equipped with a visual feedback which allows addressing even complex tasks [33]. A drawback is that the system is stationary and restricted to planar movements.

The mPower 1000 (Myomo, Inc. Cambridge, MA) is an example for an active elbow orthosis system with one degree of freedom, which is based on technology developments from MIT. The device supports the elbow movement in extension and flexion and is designed for home and clinical use. It supports patients in their rehabilitation process who suffer from the consequences of stroke, spinal cord injury, or multiple sclerosis. The system is controlled by residual signals 
of the biceps and triceps with three possible support levels [34].

Regardless of the type of rehabilitation device, most systems own one of the three basic classes of rehabilitative control strategies. These control strategies are referred to as passive-assistance, assist-as-needed, and challenge-based [35]. Focus of current research is mainly, on assist-as-needed techniques, which support the user only as much as it is necessary [36]. Within this context there is even the possibility to act against the user's movements and thus to challenge the patient during task execution [35].

Another device group compensates physical limitations in daily life of patients. A representative example of these systems is the commercially available Wilmington Robotic Exoskeleton (WREX), which relieves the weight of the arm by elastic slings. Thus, the user is able to operate with exclusion of gravity [37].

The project Ortho Jacket of the Karlsruhe Institute of Technology (KIT) has the aim to develop an active orthosis for patients with spinal marrow lesion in cervical vertebras (C4 to C7). The orthosis is intended to enable movements of the shoulder, elbow, and hand, in conjunction with a wheelchair. The control of the individual degrees of freedom is carried out via a joystick or via residual EMG signals of shoulder and arm muscles [38].

The WOTAS orthosis is able to reduce symptoms of tremor in the upper extremity. The orthosis has three active degrees of freedom. With help of gyroscopes and force sensors, the system is able to discern tremor and apply force into the limb, in order to suppress it [39].

For active controlled orthosis systems different control strategies are used. In [40] an orthotic exoskeleton for the hand was controlled in terms of EMG with a threshold approach. The EMG signal from the biceps of the contralateral arm was normalized using the maximum voluntary contraction (MVC). The threshold for closing the orthotic system was set around $50 \%$ of the MVC, and when deceeding the threshold the system would open up again on its own.

In [41] a control model for a 7-DOF upper limb exoskeleton was developed based on the Hill-muscle model. This model predicts the moment in a joint based on the activations, velocity, and lengths of muscles connected to this joint. The raw EMGs are high pass filtered full wave rectified and low pass filtered. Additionally, three formulas are used to calculate the activations, velocities, and length of the involved muscles. The predicted moments are used to control the joints of the exoskeleton. In the previous work [42] the group reported that the accuracy of their model for flexion/extension of the elbow joint was $\rho=0.88$, where $\rho$ is the correlation of their predicted moment compared to the reference moment.

In [43] a muscle model predicting the force produced by the triceps muscles under varying electrical stimulation is presented. The authors use a nonlinear Hammerstein structure for the models. They compare two parameter adaptation algorithms, the Recursive Least Squares (RLS) and an adapted online version called Alternately Recursive Least Squares (ARLS). The best model fits are given as -10.02 for RLS and 87.92 for ARLS.
Wearable haptic interfaces for use in a virtual environment, for example, rehabilitation, sport, or teleoperation tasks, are developed in $[44,45]$ and in other research projects. These portable exoskeleton systems can offer by parallel kinematics, targeted force-feedback, and visual integration a comprehensive virtual immersion within the application scenario.

\section{Active Orthosis}

In order to reach the goals in rehabilitation described in Section 1 and to study and transfer first rehabilitation concepts to exoskeleton technology, a demonstrator, which is presented in this section, was designed.

3.1. Application Scenario. The therapeutic goal of the orthosis is recovery of lost motor functions of the upper extremity after neurological diseases.

The idea is to use the device in the early stage of treatment to passively move the patient's arm. With therapy in advanced stages the residual muscle activity will be measurable again. This low residual activity may not be sufficient for moving the arm but may result in myoelectric signals. By measuring these signals with EMG, they can be used to detect the patient's movement intent.

Further, these signals can be used to move the patient's arm in a self-motivated way. In later stages of treatment the patient should regain more and more muscle strength. Therefore, the orthosis has to adjust its assistance level via the measured muscle activity, in a way that higher muscle activity leads to a lower level of assistance.

The device can enable patients to perform the following exercise modalities (which are based on the established and evidence-based rehabilitation methods).

(i) Early and Intensive Practice. Start of the arm rehabilitation, for example, few days after acute stroke with a high intensity, when indicated.

(ii) Repetitive Practice. Repetitive target movements across various sequences.

(iii) Task-Oriented Training. Exercise oriented on everyday life situations, for example, in an exercise kitchen.

(iv) Independent Training. Therapeutic treatments with intermittent supervision by the therapist.

The goal of the orthosis system is to achieve a therapy session comparable to a guided session by a therapist, without having him at site, and to motivate the patient for a constant training. Figure 2 shows a possible training session which deals with different movement patterns.

3.2. Mechanical Design. The current version of the system has one active degree of freedom and four passive joints that are required to compensate misalignments and one actuated joint to support the flexion/extension movement of the elbow joint (see Figure 3). The active joint is driven by a $24 \mathrm{~V}$ Maxon Amax 22 DC motor with a 333:1 Maxon planetary gear and a $4: 1$ worm wheel gear. For a natural force interaction, safety 


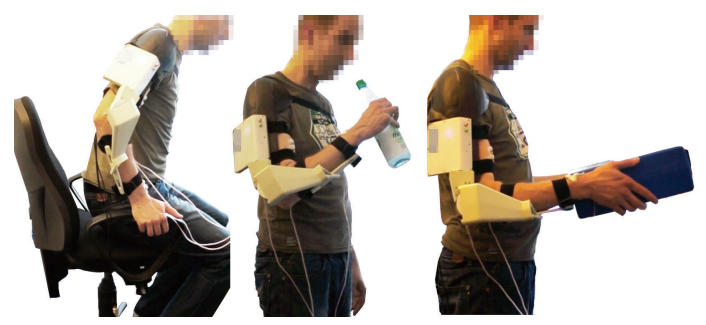

FIGURE 2: The patient is able to train independently various types of exercise of daily life. A special shoulder strap increases the stable seating of the orthosis, resulting in a better weight distribution.

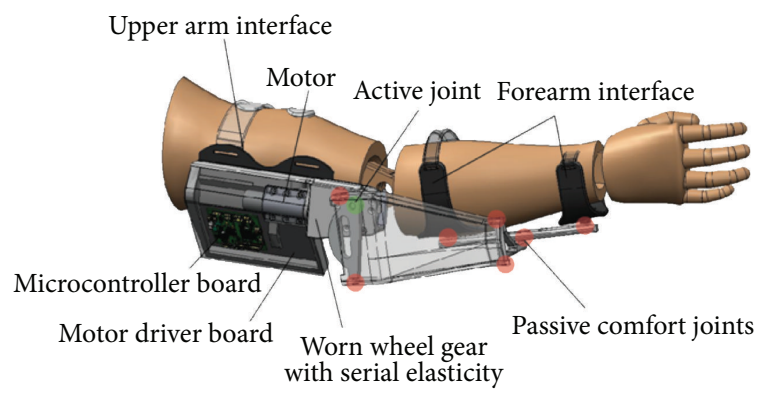

Figure 3: Mechanical design of the active orthosis. The red dots represent the positions of the passive degrees of freedom.

reasons and to measure the applied force interaction and the actuated joint is compliant. This compliance is generated via serial elasticity in the worm wheel gear setup. The worm is axial moveable and centred in the gear via disc springs. In case a load is applied, the worm is pushed to one side and, thus, the spring is compressed on this side. The position of the worm wheel is measured with a Balluff inductive sensor. In this way the applied load can be calculated. The position of the joint is measured with an IC-Haus-MH position encoder.

Furthermore, the used electronics consist of a STM32F103VE microcontroller, offering several data acquisition (GPIO) and communication (USART, CAN-bus) ports, and a BD6232 custom made PWM H-Bridge driver. The used DC-drive can generate a torque of about $16 \mathrm{Nm}$.

To avoid any danger for the user, various safety aspects are considered. To this end, the orthosis' working range is limited by mechanical stops. Furthermore, at too high forces the forearm interface will release from the orthosis.

The active range of motion of the elbow orthosis corresponds to the anatomic workspace of the human joint and is individually adjustable to each subject. If the position of the joint exceeds the workspace limits defined for the user, the reference torque is automatically set to zero and the system can only be controlled via buttons.

Since an additional and unilateral load can represent a major influence on, for example, neurological patients, the orthosis' weight with respect to the user must be kept as low as possible. Therefore, the orthosis' materials are a combination of carbon reinforced plastics and polyamide $P A 6$, for a lightweight, robust, and stiff design. Additionally, a carrying system was developed, which distributes the weight

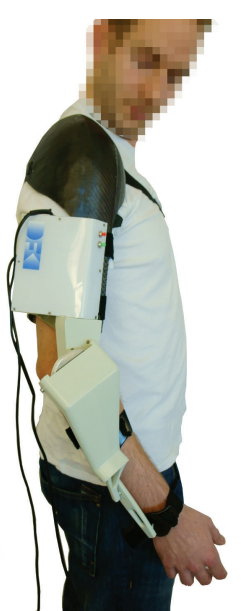

FIgURE 4: Design of the active orthosis.

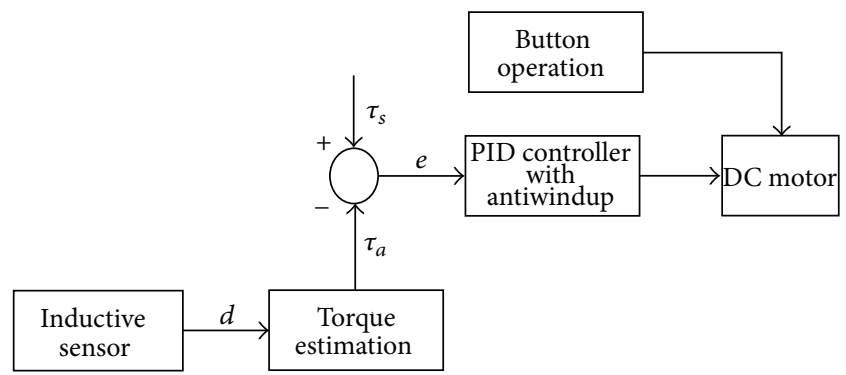

FIGURE 5: Block diagram of the torque control loop.

of the device on both shoulders. Figures 2 and 4 show the current design concept of the orthosis.

3.3. Control Architecture. Several research groups have described robotic devices for upper limb rehabilitation and their strategies to control them in a user-oriented way. In [46] the torque applied to the elbow joint of an upper extremity exoskeleton is measured via a load cell, while the set torque is calculated via muscle models. In a second step, the authors derive four performance indices, in order to calculate the magnitude of support by the exoskeleton from EMG data. In [47] an impedance control scheme is implemented. Two load cells in series estimate the joint torque which is fed into a dynamic impedance function.

In the following, the torque control system of the proposed active orthosis will be presented. This can be visualized in the simplified block diagram in Figure 5. Compared to the systems mentioned above, the torque that is applied to the orthosis' joint is measured making use of the compliance in the joint itself, as it will be further explained below.

The general control structure is designed to be cascaded, while the main and inner loop of the control architecture is a torque control loop. The DC-drive of the device is provided with two disc springs performing the serial elasticity of the drive. These springs deflect when load is applied to the joint. One is used for movements that are directed upwards and one for movements that are directed downwards. The inductive 
sensor detects this deflection $d$. With these measurements it is possible to obtain a nearly linear function between the spring deflection and the actual torque applied to the joint, $\tau_{a}$. A feature of this method is that it gives an accurate measurement for closed-loop actuator force/torque control without the need for calculating or measuring the armature current of the motor [48]. The set (desired) joint torque $\tau_{s}$ is fed externally via USART port. At this time the reference values are defined in a common PC, and later $\tau_{a}$ will be calculated online using dynamic models presented in Section 5. The difference between these two torques is the control error $e$, which is propagated into an antiwindup PID controller. The performance of the control system was verified with weight discs in order to simulate values for $\tau_{a}$, giving the corresponding $\tau_{s}$ to the system and resulting in an accurate balancing of the weights. Furthermore, the resulting measured torque was compared with the deflection-torque curve depicted above.

Alternatively, the orthosis can be manually operated via two buttons at any time, supplying a constant voltage of $\pm 15 \mathrm{~V}$. This allows corrections and repositioning of the joint if needed.

\section{Material and Methods}

4.1. Experimental Setup. This section describes the conducted experiments. The idea behind the experiments was to obtain models that can help to study and to predict important aspects of the behavior of the active orthosis.

All experiments were performed by one subject in an upright position with EMG electrodes and orthosis equipped to the subject's right arm. A monitor on a table in front of the subject was used to give commands (flexion, relaxation, and extension). This stimulation was implemented with the Presentation software (Neurobehavioral Systems, Inc., Albany, USA). The given commands were marked in the measured EMGs. We designed three different experiments which are explained in the following part.

Triggering the Orthosis. For this experiment the orthosis was fixed in different positions in a range of $[90, \ldots, 180]$ degrees in 10 degrees steps. In these positions the user performed three different actions: flexion, relaxation, and extension of the arm. Each action period took $5 \mathrm{~s}$. The routine started with a relaxation phase. Afterwards two alternating action combinations ((1) flexion/relaxation (2) extension/relaxation) were executed 10 times each. The experiments were recorded separately for each angle starting at 180 degrees. Between two measurements a short break of 2 minutes was given to the subjects.

This experiment was conducted to record data to build a model that enables triggering the movement direction which was intended by the user and is supported by the orthosis as well as the relaxation phase where the orthosis stays in a fixed position. The different starting positions were used, since in each position the muscles are contracted to a different amount, which leads to diverse signal shapes.

Level of Assistance (Flexion). For this experiment the orthosis was operated in free-run mode. The operator had to lift weights in the range of $[1, \ldots, 4] \mathrm{kg}$ in $1 \mathrm{~kg}$ steps. The experiment started with the user's arm being fully extended. The subject had to flex and extend his arm for 10 times, each action period was again $5 \mathrm{~s}$. The experiments were recorded separately for each weight starting with the lightest. Between two measurements again a short break of 2 minutes was given to the subjects.

This experiment was conducted to formalize a model which can modulate the level of assistance provided by the orthosis in dependence of the weight the operator has to lift.

Level of Assistance (Flexion and Extension). In contrast to the previously explained experiment, here forces were applied in both movement directions. The force was directly provided by the orthosis in a range of $[-2.0, \ldots, 2.0] \mathrm{Nm}$ in $0.5 \mathrm{Nm}$ steps. Note that a negative torque extends and a positive torque flexes the orthosis and a torque of zero is complementary to the free-running mode.

This experiment was conducted to formalize a model which can modulate the level of assistance provided by the orthosis in dependence of induced force and movement direction.

4.2. Acquisition and Processing of EMG and Orthosis Data. This section describes how muscle activity was measured and processed in order to use it as a control signal for the orthosis. Since the orthosis is designed to actively flex and extend the operators forearm, EMGs were measured at the biceps and triceps, which are the muscles mainly involved in the process of flexion and extension. Ag/ Ag-Cl electrodes were placed in a bipolar arrangement on the middle of the muscles in direction of the muscle fibers. The signals were acquired with a sampling frequency of $1000 \mathrm{~Hz}$ using a BrainAmp ExG MR amplifier (Brain Products GmbH, Gilching, Germany).

The EMGs were preprocessed in two consecutive steps. First a variance based filter [49] was applied. This filtering step eliminates motion artifacts and enhances the signal-to-noise ratio of EMG signals. The length of the filter was set to $50 \mathrm{~ms}$. Basically a sliding window of length $50 \mathrm{~ms}$ is passed to the signal with a step width of $1 \mathrm{~ms}$. The variance of the whole window is assigned as the new value of the last sample inside the window, resulting in the filtered signal. In a second step the Root Mean Square (RMS) of the signal was calculated. For the calculation, again a window of $50 \mathrm{~ms}$ was used, but in this case the step width was chosen as $50 \mathrm{~ms}$ so that the windows did not overlap. The resulting signal had a frequency of $20 \mathrm{~Hz}$. In this way we obtained the same sampling frequency as the sensors of the orthosis.

In addition to the EMGs, we recorded sensor values from the orthosis. These are the position and the force induced into the orthosis by the operator's arm. The signals were acquired with $20 \mathrm{~Hz}$ and sent via RS-232 to a computer where they were stored. In order to synchronize the EMG and sensor data, we marked the beginning and end of the orthosis measurements in the EMG. Since the preprocessed EMGs have the same sampling frequency as the orthosis sensor data, the corresponding EMG part could be cut and merged into one time series together with the sensor data. The processing was done with MATLAB 2009a (The MathWorks 
Inc., Natick, USA). For loading the EMG data the EEGLAB toolbox (Swartz Center for Computational Neuroscience, San Diego, USA) was used.

4.3. System Identification with the RLS Algorithm. In the field of system identification the Recursive Least Squares (RLS) method is a basic estimation method [50]. Its principle is simple and relatively easy to use. In most of the cases the algorithm delivers high accuracy, fast convergence of parameters, and high modelling efficiency. What also makes this algorithm attractive is the fact that it can easily be extended for identification of more complex and nonlinear models.

Let $t$ be the actual time step. The general structure of the RLS algorithm is given by

$$
\widehat{\theta}(t+1)=\widehat{\theta}(t)+F(t+1) \phi(t) \varepsilon^{0}(t+1)
$$

with the adaptation gain:

$$
F(t+1)=F(t)-\frac{F(t) \phi(t) \phi(t)^{T} F(t)}{1+\phi(t)^{T} F(t) \phi(t)}
$$

and the prediction error

$$
\varepsilon^{0}(t+1)=y(t+1)-\widehat{\theta}(t)^{T} \phi(t) .
$$

$\widehat{\theta}$ is the vector of computed model parameters.

Furthermore $\phi(t)$ is the predictor regressor vector or the vector of measurable signals, in which the real input values $u(t)$ and the real output values $y(t)$ are fed:

$$
\phi(t)^{T}=[-y(t), u(t)]
$$

Finally, the model output $\hat{y}$ is computed as follows:

$$
\widehat{y}(t+1)=\widehat{\theta}(t+1)^{T} \phi(t) \text {. }
$$

It is common to provide the adaptation gain with a forgetting factor, in order to improve performance.

This method is widely used in engineering for identification of several dynamic systems, for example, electromotors. For a more detailed description and an example of the use of this algorithm for identification of a nonlinear model, please see [51].

Using our own MATLAB implementation of the RLS algorithm, and following the experiment descriptions in Section 4.1, models of important dynamic relationships of the active orthosis were identified. The parameters of the models were adapted, in specific, with (1). The motivations for the identification of the models presented in the following paragraphs were that particularly in rehabilitation, on the one side, the need for an accurate discrimination of movement direction is of major importance and, on the other side, the requirement of a correct computation of the level of support currently needed by the user, according to all measurable states of the system, including muscular states.

Multi-input, single output (MISO) model structures were chosen for identification. The learning set always consisted

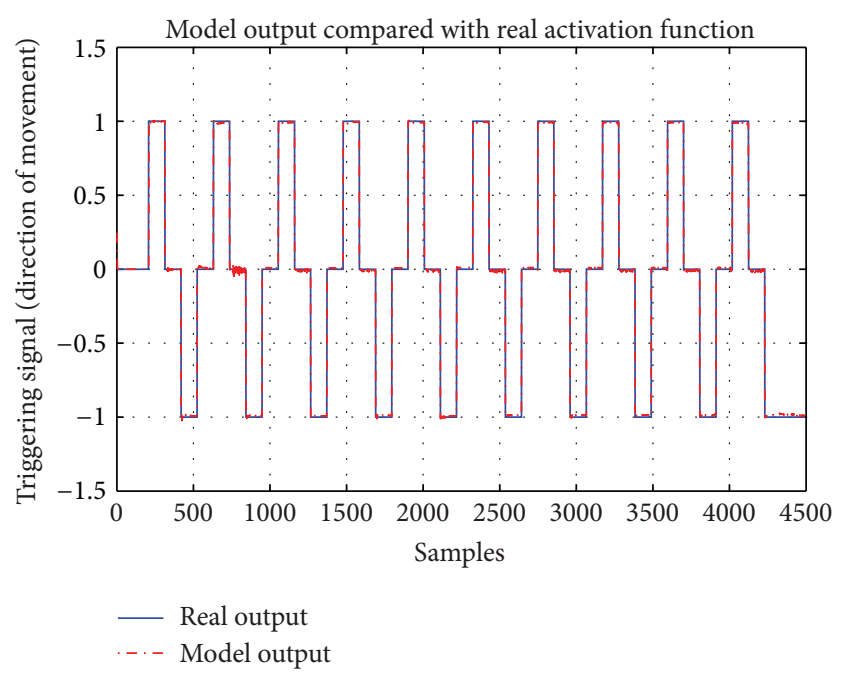

FIGURE 6: Modelling of the activation function of the orthosis. The real function (blue) is compared with the modelled function (red). Model fit reaches over 99\%. In this example the orthosis was fixed at $90^{\circ}$ angular position.

of the first half of samples available and the validation set of the second half. Both muscle (EMG) and device information were used. The four inputs of the model are the preprocessed EMGs of the triceps and biceps $\mathrm{EMG}_{\text {tri }}$ and $\mathrm{EMG}_{\mathrm{bi}}$, the torque applied on the orthosis' joint measured via the displacement of a disc spring $\tau_{\text {spring }}$, and the angular position values of the orthosis $\theta$.

\section{Results}

In this section the experimental results are presented. First, the triggering function of the orthosis, which defines when and in which direction the system should move, was approximated. Consider the following:

$$
\begin{aligned}
y 1_{\text {mod }}= & \frac{-3.2251 \cdot 10^{-7} z+6.5147 \cdot 10^{-8}}{z^{3}-0.9717 z^{2}-0.0163 z-0.001} \cdot \mathrm{EMG}_{\text {tri }} \\
& +\frac{-2.0212 \cdot 10^{-6} z+5.3232 \cdot 10^{-7}}{z^{3}-0.9717 z^{2}-0.0163 z-0.001} \cdot \mathrm{EMG}_{\mathrm{bi}} \\
& +\frac{0.0135 z-0.0104}{z^{3}-0.9717 z^{2}-0.0163 z-0.001} \cdot \tau_{\text {spring }} \\
& +\frac{-1.9671 \cdot 10^{-6} z+1.2784 \cdot 10^{-6}}{z^{3}-0.9717 z^{2}-0.0163 z-0.001} \cdot \theta
\end{aligned}
$$

The resulting model of the triggering function $y 1$ mod, given by (6), fits the real triggering function excellently (mean absolute error less than 0.001) and can be seen in Figure 6. Here, a value of -1 represents a triggering of an extension movement, a value of 1 represents a triggering of a flexion movement, and a value of 0 corresponds to the resting state (no triggering). One can see, from these figures, that the biggest model errors are reached at the phases with no active movement. We assume this is due to sensor noise in the used magnetic position encoder. 


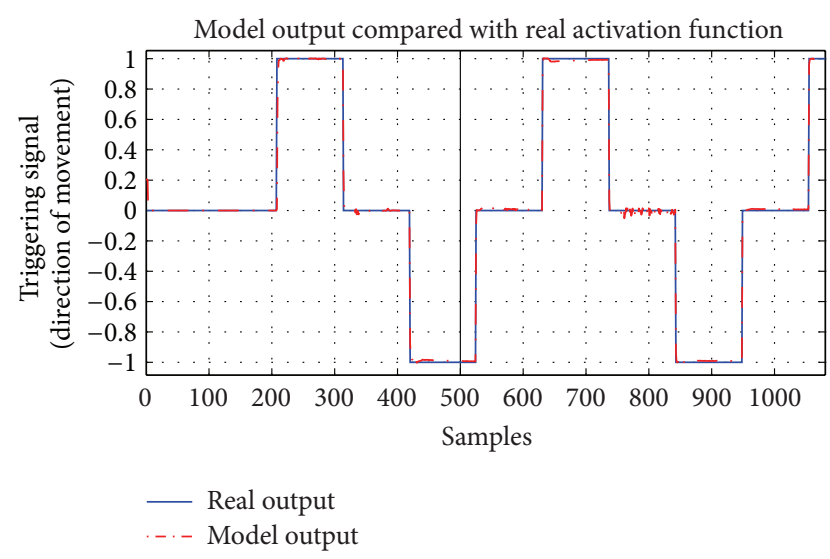

FIgURE 7: Modelling of the activation function of the orthosis. The precision of the model can be seen in this enlargement.

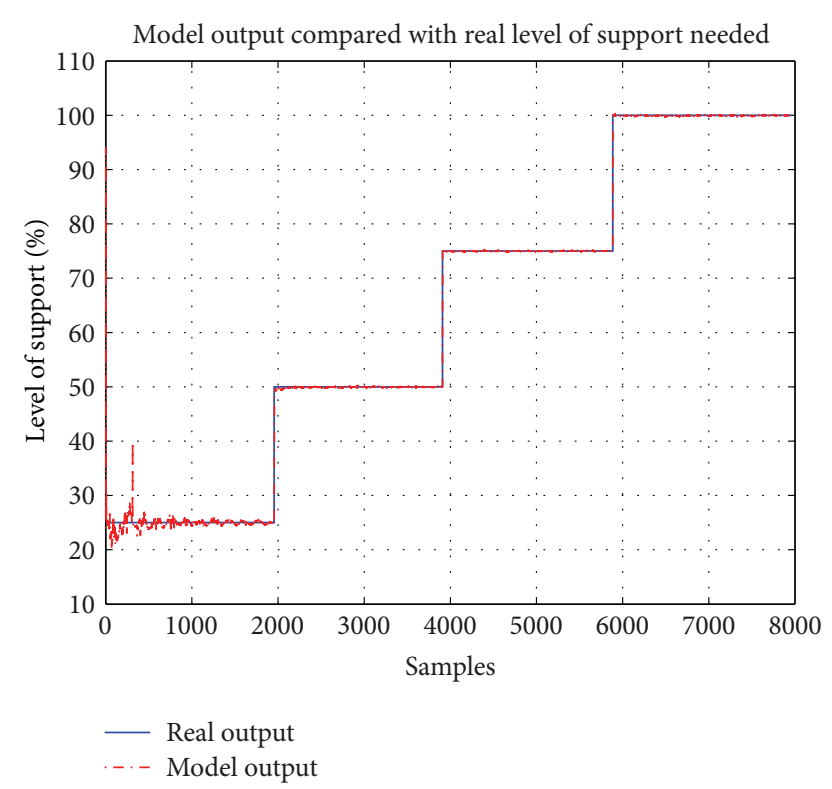

FIGURE 8: Modelling of the support function of the orthosis, experiment 1 . The level of support is only calculated for the upward direction.

For better visualization, an enlarged fragment of the results can be seen in Figure 7. At about 750 samples the model output shows a bigger noise component. At this point at least one of the muscles could not reach the relaxation state entirely. Even in this situation the model handles the signals in an acceptable way.

Further, a second model that can help to determine the level of support needed from the orthosis was identified. The input signals chosen are the same as in the first model. In order to determine the real level-of-assistance function, in a first step experiments were performed with different external weights carried with the hand while moving the arm with the device (see Section 4.1).

The drawback of this experimental setup is that it is almost only useful to obtain biceps data, since the counterforce exerted by the weights is always acting in one

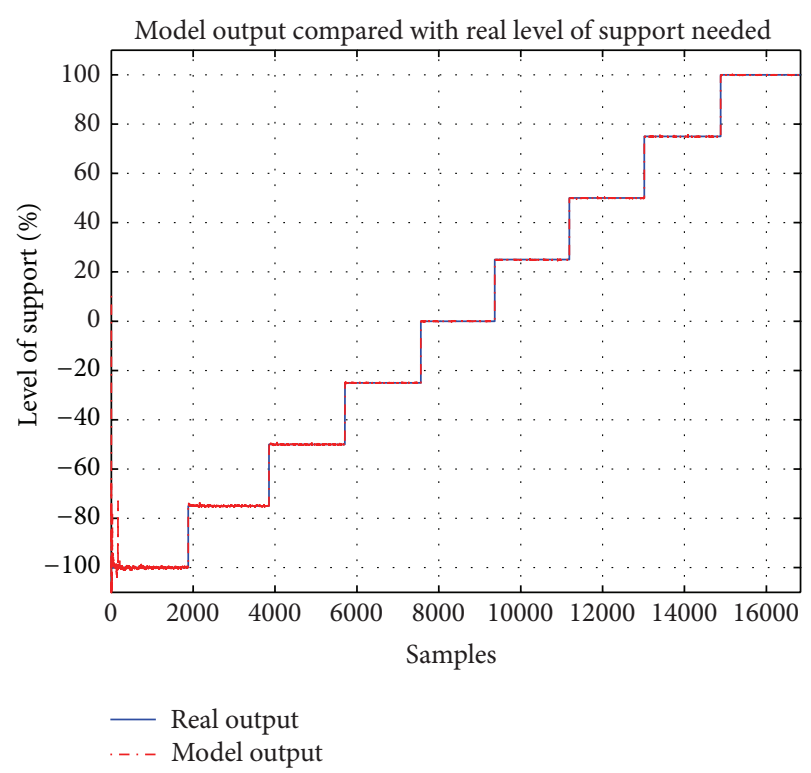

FIGURE 9: Modelling of the support function of the orthosis. The real function (blue) is compared with the modelled function (red, dotted). Model fit reaches over 99\%.

direction (Figure 8). That is the reason why in a second step it was decided to generate counterforces in both up and down directions (and, thus, to compute the level of support in both directions) over the orthosis' torque control loop, obtaining again excellent results. Here, $100 \%$ level of support corresponds to $2 \mathrm{Nm}$ and $-100 \%$ level of support corresponds to $-2 \mathrm{Nm}$. Consider the following:

$$
\begin{aligned}
y 2 \bmod = & \frac{1.0278 \cdot 10^{-8} z+7.4272 \cdot 10^{-9}}{z^{3}-0.8757 z^{2}-0.1104 z-0.0138} \cdot \mathrm{EMG}_{\mathrm{tri}} \\
& +\frac{1.0294 \cdot 10^{-7} z-1.0769 \cdot 10^{-7}}{z^{3}-0.8757 z^{2}-0.1104 z-0.0138} \cdot \mathrm{EMG}_{\mathrm{bi}} \\
& +\frac{0.002 z-0.0029}{z^{3}-0.8757 z^{2}-0.1104 z-0.0138} \cdot \tau_{\text {spring }} \\
& +\frac{-4.7578 \cdot 10^{-5} z+4.6896 \cdot 10^{-5}}{z^{3}-0.8757 z^{2}-0.1104 z-0.0138} \cdot \theta .
\end{aligned}
$$

The resulting model of the support function $y 2 \bmod$ is given by (7). The performance of the modelling algorithm can be seen in Figure 9. One can see that the prediction error is bigger at the beginning of the measurements. These are the first samples of the learning set and one can observe the modelling algorithm converging after 2000 samples. This initial error could probably be reduced with further fine-tuning of initial model parameters. Throughout the validation set, only isolated deviations, which are not significant (caused by sensor noise), can be seen.

The precision of the model can be better observed in the enlargement shown in Figure 10.

In both experiments a high model fit could be reached. A big contribution to that is the fact that the measurable signals 


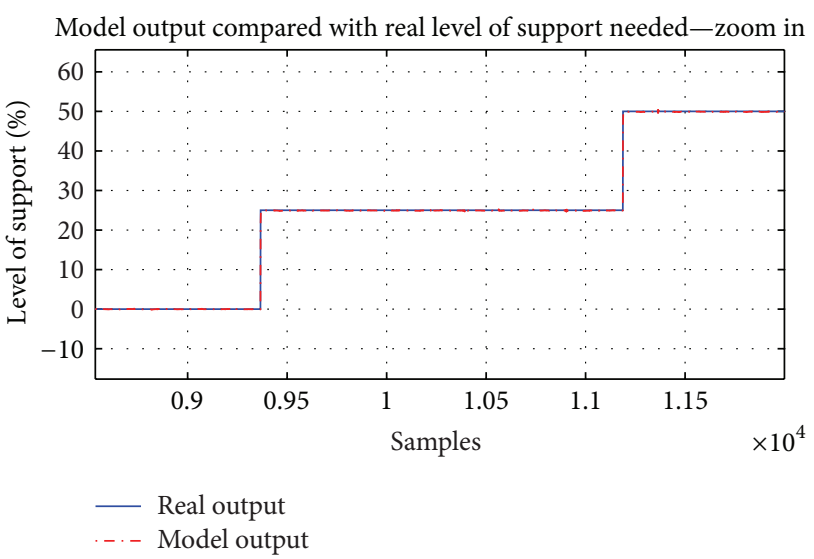

FIGURE 10: Modelling of the support function of the orthosis, enlarged view.

used to identify the models, especially the EMG signals, were properly preprocessed, so that it was possible to work with largely clean signals. This is very important, since the RLS algorithm reacts sensitively to noise contaminated signals. Furthermore, the high model fit is also an indicator of a strong correlation between the inputs and the desired output signal. Compared to the Hill model in [46] the proposed blackbox model is significantly easier to formalize mainly in two aspects. The first one is the preprocessing of the myosignals since the used here variance filter works in one step compared to a two-filter preprocessing. The other point is that for the formalization of the models presented in this paper the signals are directly fed into the identification algorithm, while in [46] important variables, for example, the length of the muscles, have to be computed first in some steps before computing the output of the model.

Compared to the work presented in [43], in which the RLS algorithm compared to an adapted form of the same is used for identification, our proposed model structure is simpler, since we have formalized linear models which generally have a lower computational complexity than (Hammerstein) nonlinear models. Further, it has to be stressed that the experimental setup used in [43] is different, since electrical stimuli given to a fixed upper extremity were measured instead of EMG, while the torque is measured via a force/torque sensor installed at a padded hand grip.

Nevertheless, in contrast to the two above-mentioned studies, where the torque produced by the muscles was computed, in the present work, control signals as well as the torque needed for assisting the user were modelled.

\section{Conclusions and Outlook}

In this work we presented some background information and facts that support the use of robotic systems for rehabilitation processes. Further, an overview of exoskeleton technology was given. Starting from this knowledge, concepts on the feasibility of exoskeleton technology for home rehabilitation were given and discussed by means of a demonstrator, an active 1-DOF-elbow orthosis. Its possible application, design and mechanics, and control were presented. To summarize, the system allows supporting self-initiated movements that are normally executed by both upper arm muscles M. biceps brachii or M. triceps brachii.

Two dynamic models, identified with the RLS algorithm, were shown. The first one computes the triggering function of the system from the muscle and sensor information from the orthosis, while the second one computes a function describing the level of support needed to counteract external forces. Both models show excellent performance in matching the real signals.

The next step is to integrate the obtained models into the orthosis' control system, turning it into a model-based control scheme. It is expected that with the predicted triggeringand torque-assistive function the control system of the active orthosis will improve in two points: in the discrimination of time and direction of movements and on the other side in the precision at defining proper assistive set torque values, according to the current state of all measurable signals in the system.

The derived models are patient and session-specific and therefore have to be trained individually. EMGs of a single subject may vary from session to session, due to slightly different electrode positions or resistances and due to different levels of muscle fatigue. Effects between subjects are likely even bigger, since the physical conditions of the muscles are completely different.

Nevertheless, in the future we will investigate the transferability of a model from one subject to another or work into the direction of adaptive models. Further, the model reliability for more natural movements will be evaluated. It can be expected that the parameters of the presented models derived with data acquired under a controlled setting will vary for natural movements. However, the model structure might remain the same. Follow-up experiments with daily life task, for example, grasping a glass, getting up from a chair, or lifting items, are planned to verify this.

Nonetheless, the presented results are very promising, in terms of the control accuracy of orthotic systems driven by myoelectric signals and sensor data from the device itself. In particular, the automatic adjustment of assistance regulated by the means of EMG leads into the direction of an autonomous assist-as-needed home rehabilitation system.

Finally, the design of the next version of the system is currently under development. From the electromechanical point of view it is intended to use a brushless DC (BLDC) motor in combination with a harmonic drive. Advantages of harmonic drives are among others high torque capacity with compact and lightweight designs, as well as zero backlash. In this case the torque will be measured via the motor's current. An important point will be to give some kind of feedback to the user on the effects of the rehabilitation process.

In order to give the system full mobility, the power will be supplied via battery packs, which will be installed in a decentralized module that may be carried at the lower back of the user. This is located close to the human center of gravity and, therefore, minimizes the load that is put on the user.

One possibility of a redesign of the system can be seen in Figure 11. 


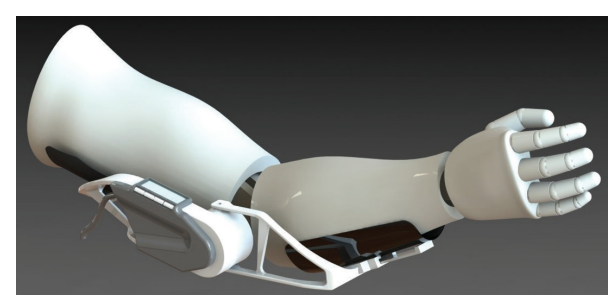

FIGURE 11: One possible design for the future version of the active orthosis.

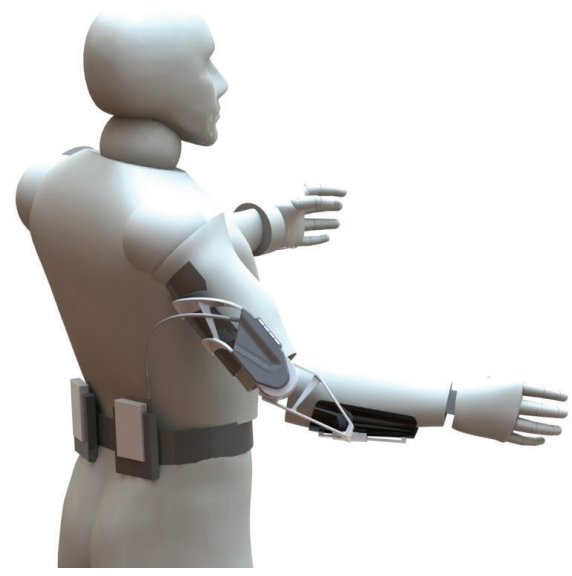

FIGURE 12: One possible redesign for the future version of the active orthosis-back perspective.

Figure 12 shows the design from the back perspective, including the battery packs.

\section{Conflict of Interests}

The authors declare that there is no conflict of interests regarding the publication of this paper.

\section{Acknowledgments}

This work was supported by the German Bundesministerium für Bildung und Forschung (BMBF, Grant FKZ 01IW10001) and the German Bundesministerium für Wirtschaft und Technologie (BMWI, Grant FKZ 50 RA 1011).

\section{References}

[1] C. Fuchs, "Demografischer wandel und notwendigkeit der priorisierung im gesundheitswesen. Positionsbestimmung der Ärzteschaft," Bundesgesundheitsbl Gesundheitsforsch Gesundheitsschutz, vol. 83, pp. 435-440, 2010.

[2] H. I. Krebs, J. J. Palazzolo, L. Dipietro et al., "Rehabilitation robotics: performance-based progressive robot-assisted therapy," Autonomous Robots, vol. 15, no. 1, pp. 7-20, 2003.

[3] M. J. H. Lum, D. Trimble, J. Rosen et al., "Multidisciplinary approach for developing a new minimally invasive surgical robotic system," in Proceedings of the 1st IEEE/RAS-EMBS International Conference on Biomedical Robotics and Biomechatronics (BioRob '06), pp. 841-846, Tuscany, Italy, February 2006.
[4] S. Hesse, H. Schmidt, C. Werner, and A. Bardeleben, "Upper and lower extremity robotic devices for rehabilitation and for studying motor control," Current Opinion in Neurology, vol. 16, no. 6, pp. 705-710, 2003.

[5] R. C. V. Loureiro, W. S. Harwin, K. Nagai, and M. Johnson, "Advanahces in upper limb stroke rehabilitation: a technology push," Medical \& Biological Engineering \& Computing, vol. 49, no. 10, pp. 1103-1118, 2011.

[6] H. Masur, "Sinnvoller einsatz von robotern in der neurorehabilitation-fiktion oder realität?” Deutsches Ärzteblatt, vol. 105, no. 18, p. 329, 2008.

[7] T. Henze, "Moderne rehabilitation nach schlaganfall," NeuroTransmitter, no. 10, pp. 59-67, 2007.

[8] E. A. Kirchner, J. C. Albiez, A. Seeland, M. Jordan, and F. Kirchner, "Towards assistive robotics for home rehabilitation," in Proceedings of the 6th International Conference on Biomedical Electronics and Devices (BIODEVICES '13), pp. 168-177, SciTePress, Barcelona, Spain, 2013.

[9] L. M. V. Benitez, N. Will, M. Tabie, S. Schmidt, E. Kirchner, and J. Albiez, "An EMG-based assistive orthosis for upper limb rehabilitation," in Proceedings of the International Conference on Biomedical Electronics and Devices (BIODEVICES '13), pp. 323328, SciTePress, Barcelona, Spain, February 2013.

[10] C. Deaton, E. S. Froelicher, L. H. Wu, C. Ho, K. Shishani, and T. Jaarsma, "The global burden of cardiovascular disease," European Journal of Cardiovascular Nursing, vol. 10, supplement 2, pp. S5-S13, 2011.

[11] S. J. Albert and J. Kesselring, "Neurorehabilitation of stroke," Journal of Neurology, vol. 259, no. 5, pp. 817-832, 2012.

[12] J. H. Cauraugh and J. J. Summers, "Neural plasticity and bilateral movements: a rehabilitation approach for chronic stroke," Progress in Neurobiology, vol. 75, no. 5, pp. 309-320, 2005.

[13] S. Hesse, C. Werner, and J. Brocke, "Use of machines and robots in neuro rehabilitation expectations of the clinics," OrthopädieTechnik, vol. 2, pp. 74-77, 2009.

[14] P. Frommelt and H. Lösslein, Neuro Rehabilitation, Springer, Berlin, Germany, 2010.

[15] H. C. Diener, W. Hacke, and M. Forsting, Eds., Schlaganfall, Georg Thieme, Berlin, Germany, 2004.

[16] K. Müller, C. M. Bütefisch, R. J. Seitz, and V. Hömberg, "Mental practice improves hand function after hemiparetic stroke," Restorative Neurology and Neuroscience, vol. 25, no. 5-6, pp. 501511, 2007.

[17] J. H. Morris, F. van Wijck, S. Joice, S. A. Ogston, I. Cole, and R. S. MacWalter, "Bilaterales oder unilaterales training der oberen extremität nach schlaganfall? Eine randomisierte kontrollierte studie," Physioscience, vol. 4, no. 4, pp. 194-195, 2008.

[18] C. J. Winstein, D. K. Rose, S. M. Tan, R. Lewthwaite, H. C. Chui, and S. P. Azen, "A randomized controlled comparison of upper-extremity rehabilitation strategies in acute stroke: a pilot study of immediate and long-term outcomes," Archives of Physical Medicine and Rehabilitation, vol. 85, no. 4, pp. 620-628, 2004.

[19] C. Dohle, J. Püllen, A. Nakaten, J. Küst, C. Rietz, and H. Karbe, "Mirror therapy promotes recovery from severe hemiparesis: a randomized controlled trial," Neurorehabilitation and Neural Repair, vol. 23, no. 3, pp. 209-217, 2009.

[20] B. R. Brewer, S. K. McDowell, and L. C. Worthen-Chaudhari, "Poststroke upper extremity rehabilitation: a review of robotic systems and clinical results," Topics in Stroke Rehabilitation, vol. 14, no. 6, pp. 22-44, 2007. 
[21] L. Pignolo, "Robotics in neuro-rehabilitation," Journal of Rehabilitation Medicine, vol. 41, no. 12, pp. 955-960, 2009.

[22] G. B. Prange, M. J. A. Jannink, C. G. M. Groothuis-Oudshoorn, H. J. Hermens, and M. J. Ijzerman, "Systematic review of the effect of robot-aided therapy on recovery of the hemiparetic arm after stroke," Journal of Rehabilitation Research and Development, vol. 43, no. 2, pp. 171-184, 2006.

[23] S. Hesse, J. Mehrholz, and C. Werner, "Roboter-und gerätegestützte rehabilitation nach schlaganfall," Deutsches Ärzteblatt, vol. 105, no. 18, pp. 330-336, 2008.

[24] S. J. Housman, K. M. Scott, and D. J. Reinkensmeyer, "A randomized controlled trial of gravity-supported, computerenhanced arm exercise for individuals with severe hemiparesis," Neurorehabilitation and Neural Repair, vol. 23, no. 5, pp. 505514, 2009.

[25] T. Nef, G. Colombo, and R. Riener, "ARMin—robot for movement therapy of the upper extremities," Automatisierungstech$n i k$, vol. 53, no. 12, pp. 597-606, 2005.

[26] N. Hogan, H. I. Krebs, J. Charnnarong, P. Srikrishna, and A. Sharon, "MIT-MANUS: a workstation for manual therapy and training. I," in Proceedings of the IEEE International Workshop on Robot and Human Communication (ROMAN '92), pp. 161165, Tokyo, Jaban, September 1992.

[27] P. S. Lum, M. Van der Loos, P. Shor, and C. G. Burgar, "A robotic system for upper-limb exercises to promote recovery of motor function following stroke," in Proceedings of the International Conference on Rehabilitation Robotics (ICORR '99), Stanford University, 1999.

[28] A. Mayr, M. Kofler, and L. Saltuari, "ARMOR: an electromechanical robot for upper limb training following stroke. A prospective randomised controlled pilot study," Handchirurgie Mikrochirurgie Plastische Chirurgie, vol. 40, no. 1, pp. 66-73, 2008.

[29] G. Kwakkel, B. J. Kollen, and H. I. Krebs, "Effects of robotassisted therapy on upper limb recovery after stroke: a systematic review," Neurorehabilitation and Neural Repair, vol. 22, no. 2, pp. 111-121, 2008.

[30] T. Nef, M. Mihelj, G. Kiefer, C. Perndl, R. Müller, and R. Riener, "ARMin-exoskeleton for arm therapy in stroke patients," in Proceedings of the IEEE 10th International Conference on Rehabilitation Robotics (ICORR '07), pp. 68-74, Noordwijk, Netherlands, June 2007.

[31] S. J. Housman, V. Le, T. Rahman, R. J. Sanchez Jr., and D. J. Remkensrneyer, "Arm-training with T-WREX after chronic stroke: preliminary results of a randomized controlled trial," in Proceedings of the IEEE 10th International Conference on Rehabilitation Robotics (ICORR '07), pp. 562-568, Noordwijk, Netherlands, June 2007.

[32] G. B. Prange, M. J. A. Jannink, A. H. A. Stienen, H. Van Der Kooij, M. J. Ijzerman, and H. J. Hermens, "Influence of gravity compensation on muscle activation patterns during different temporal phases of arm movements of stroke patients," Neurorehabilitation and Neural Repair, vol. 23, no. 5, pp. 478485, 2009.

[33] H. I. Krebs, B. T. Volpe, D. Williams et al., "Robot-aided neurorehabilitation: a robot for wrist rehabilitation," IEEE Transactions on Neural Systems and Rehabilitation Engineering, vol. 15, no. 3, pp. 327-335, 2007.

[34] J. Stein, K. Narendran, J. McBean, K. Krebs, and R. Hughes, "Electromyography-controlled exoskeletal upper-limb-powered orthosis for exercise training after stroke," American
Journal of Physical Medicine and Rehabilitation, vol. 86, no. 4, pp. 255-261, 2007.

[35] L. Marchal-Crespo and D. J. Reinkensmeyer, "Review of control strategies for robotic movement training after neurologic injury," Journal of NeuroEngineering and Rehabilitation, vol. 6, no. 20, 2009.

[36] A. Jackson, R. Culmer, S. Makower et al., "Initial patient testing of iPAM - a robotic system for Stroke rehabilitation," in Proceedings of the IEEE 10th International Conference on Rehabilitation Robotics (ICORR '07), pp. 250-256, Noordwijk, Netherlands, June 2007.

[37] T. Rahman, W. Sample, R. Seliktar et al., "Design and testing of a functional arm orthosis in patients with neuromuscular diseases," IEEE Transactions on Neural Systems and Rehabilitation Engineering, vol. 15, no. 2, pp. 244-251, 2007.

[38] B. Schmitz, R. Wiegand, Ch. Pylatiuk, R. Rupp, and St. Schulz, "Erste erfahrungen mit dem orthojacket," Orthopädie-Technik, vol. 4, no. 11, pp. 256-261, 2011.

[39] E. Rocon, J. M. Belda-Lois, A. F. Ruiz, M. Manto, J. C. Moreno, and J. L. Pons, "Design and validation of a rehabilitation robotic exoskeleton for tremor assessment and suppression," IEEE Transactions on Neural Systems and Rehabilitation Engineering, vol. 15, no. 3, pp. 367-378, 2007.

[40] M. DiCicco, L. Lucas, and Y. Matsuoka, "Comparison of control strategies for an EMG controlled orthotic exoskeleton for the hand," in Proceedings of the IEEE International Conference on Robotics and Automation (ICRA '04), pp. 1622-1627, New Orleans, La, USA, May 2004.

[41] J. Rosen and J. C. Perry, "Upper limb powered exoskeleton," International Journal of Humanoid Robotics, vol. 4, no. 3, pp. 529-548, 2007.

[42] E. E. Cavallaro, J. Rosen, J. C. Perry, and S. Burns, "Real-time myoprocessors for a neural controlled powered exoskeleton arm," IEEE Transactions on Biomedical Engineering, vol. 53, no. 11, pp. 2387-2396, 2006.

[43] F. Le, I. Markovsky, C. Freeman, and E. Rogers, "Online identification of electrically stimulated muscle models," in Proceedings of the American Control Conference (ACC '11), pp. 90-95, San Francisco, Calif, USA, July 2011.

[44] M. Folgheraiter, E. A. Kirchner, A. Seeland et al., "A multimodal brain-arm interface for operation of complex robotic systems and upper limb motor recovery," in Proceedings of the International Conference on Biomedical Electronics and Devices (BIODEVICES '11), pp. 150-162, Rome, Italy, January 2011.

[45] D. Tsetserukou, K. Sato, and S. Tachi, "ExoInterfaces: novel exosceleton haptic interfaces for virtual reality, augmented sport and rehabilitation," in Proceedings of the 1st Augmented Human International Conference (AH '10), H. Saito, J.-M. Seigneur, G. Moreau, and P. Mistry, Eds., April 2010.

[46] J. Rosen, M. Brand, M. B. Fuchs, and M. Arcan, "A myosignalbased powered exoskeleton system," IEEE Transactions on Systems, Man, and Cybernetics Part A, vol. 31, no. 3, pp. 210-222, 2001.

[47] D. S. Andreasen, S. K. Allen, and D. A. Backus, "Exoskeleton with EMG based active assistance for rehabilitation," in Proceedings of the IEEE 9th International Conference on Rehabilitation Robotics (ICORR '05), pp. 333-336, July 2005.

[48] D. W. Robinson, Design and analysis of series elasticity in closedloop actuator force control [Ph.D. thesis], Massachusetts Institute of Technology (MIT), Cambridge, Mass, USA, 2000.

[49] M. Tabie and E. A. Kirchner, "EMG onset detectioncomparison of different methods for a movement prediction 
task based on EMG," in Proceedings of the 6th International Conference on Bio-inspired Systems and Signal Processing (BIOSIGNALS '13), I. S. Alvarez, J. Solé-Casals, A. Fred, and H. Gamboa, Eds., pp. 242-247, SciTePress, Barcelona, Spain, February 2013.

[50] I. Landau and G. Zito, Digital Control Systems, Springer, Berlin, Germany, 2006.

[51] M. Folgheraiter, M. Jordan, L. M. V. Benitez et al., "Development of a low-pressure fluidic servovalve for wearable haptic interfaces and lightweight robotic systems," in Informatics in Control, Automation and Robotics, J. Filipe, J. A. Cetto, and J.-L. Ferrier, Eds., vol. 89 of Lecture Notes in Electrical Engineering, pp. 239253, Springer, Berlin, Germany, 2011. 

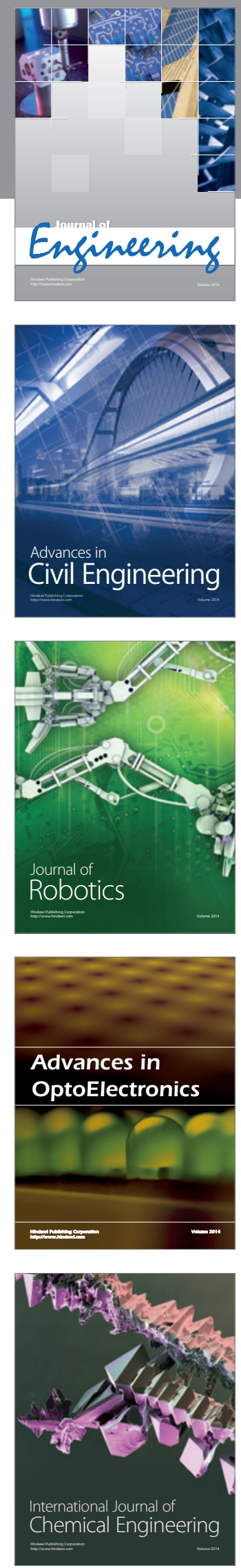

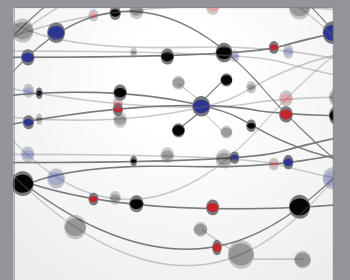

The Scientific World Journal
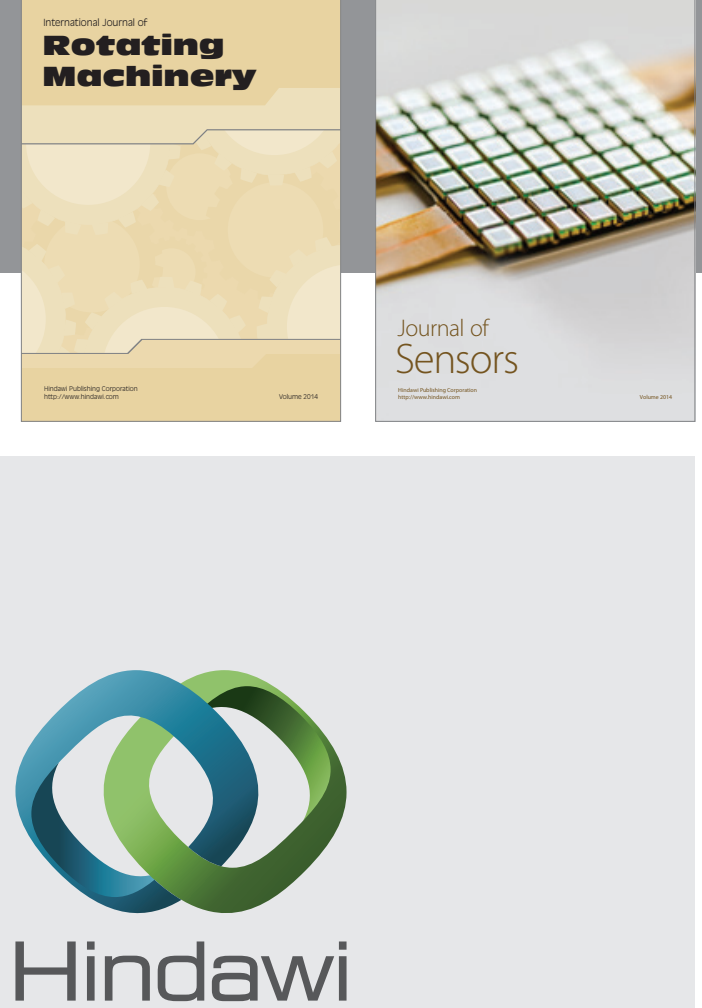

Submit your manuscripts at http://www.hindawi.com
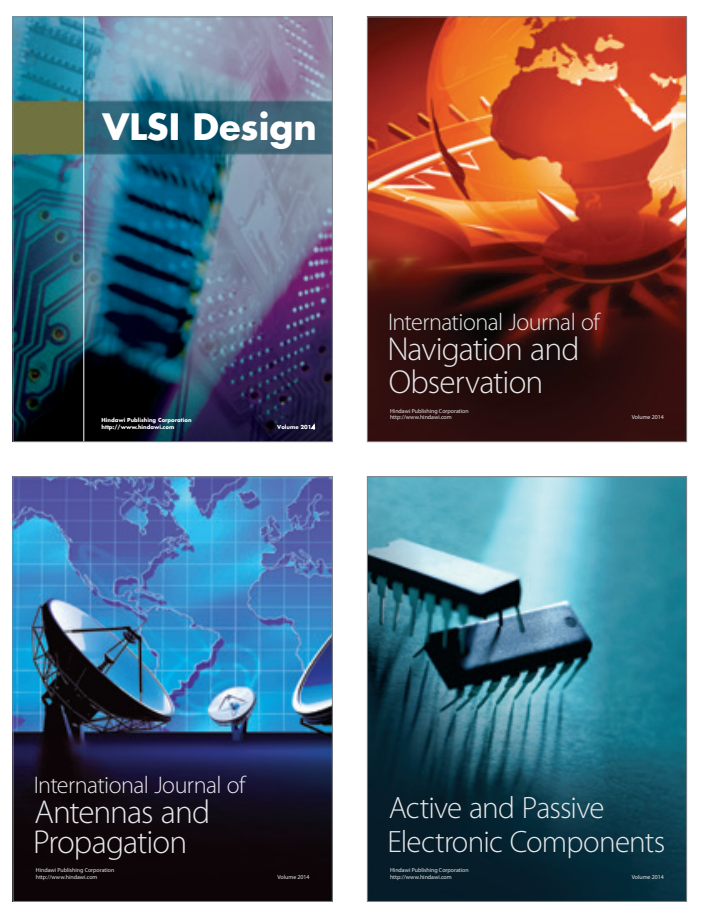
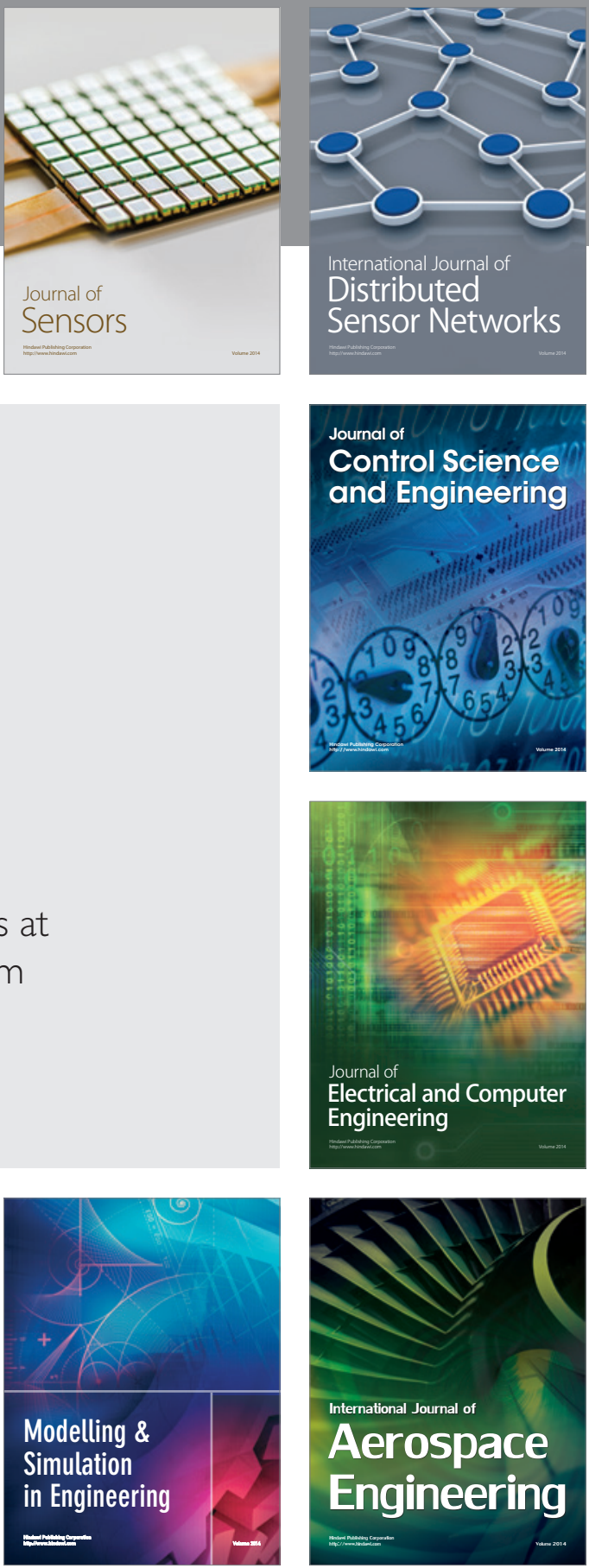

Journal of

Control Science

and Engineering
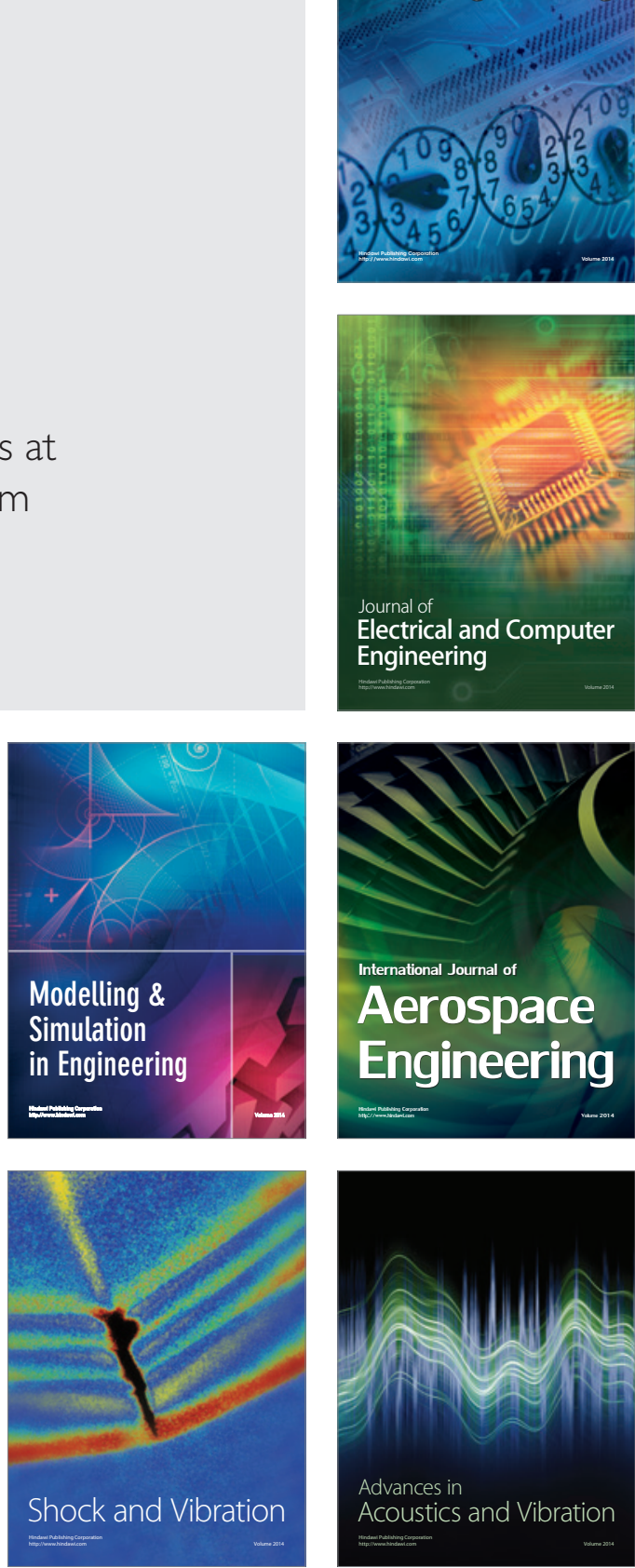